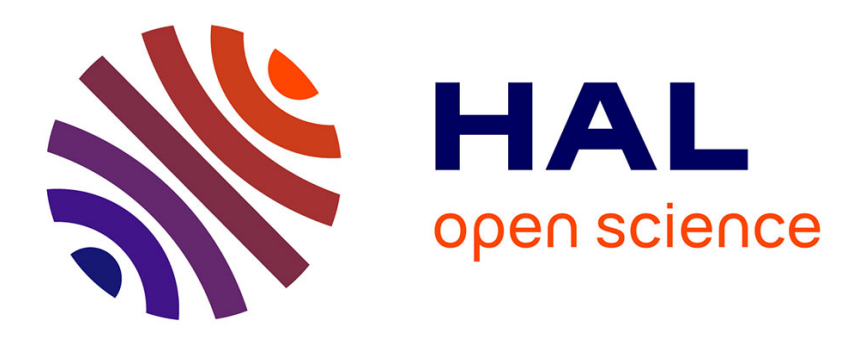

\title{
22. Étude de l'effet tunnel assisté par phonons dans des hétérojonctions p-n
}

\author{
A. Laugier
}

\section{To cite this version:}

A. Laugier. 22. Étude de l'effet tunnel assisté par phonons dans des hétérojonctions p-n. Revue de Physique Appliquée, 1970, 5 (6), pp.907-907. 10.1051/rphysap:0197000506090702 . jpa-00243492

\section{HAL Id: jpa-00243492 https://hal.science/jpa-00243492}

Submitted on 1 Jan 1970

HAL is a multi-disciplinary open access archive for the deposit and dissemination of scientific research documents, whether they are published or not. The documents may come from teaching and research institutions in France or abroad, or from public or private research centers.
L'archive ouverte pluridisciplinaire HAL, est destinée au dépôt et à la diffusion de documents scientifiques de niveau recherche, publiés ou non, émanant des établissements d'enseignement et de recherche français ou étrangers, des laboratoires publics ou privés. 


\section{2. ÉTUDE DE L'EFFET TUNNEL ASSISTÉ PAR PHONONS DANS DES HÉTÉROJONCTIONS $p$-n}

\section{A. LAUGIER}

Nous avons réalisé des hétérojonctions tunnel GeGaAs par épitaxie en phase liquide. La participation des phonons aux transitions tunnel crée des structures dépendant de la polarisation de la jonction et apparaissent par un accroissement de la résistance $\mathrm{d} V / \mathrm{d} I$ et sur $\mathrm{d}^{2} V / \mathrm{d} I^{2}$. Le comportement d'une structure $p$ Ge- $n$ GaAs est identique à celui d'une diode tunnel $p$ - $n$ GaAs. Le spectre de phonons dans $n$ Ge- $p$ GaAs est caractéristique du Ge. L'effet tunnel assisté par les phonons dans cette structure est interprété en terme de deux processus en marche à l'aide d'un état évanescent. Les caractéristiques tunnel de $p \mathrm{Ge}-n \mathrm{GaAs}$ sont interprétées par l'émission de phonons de GaAs par une excitation incohérente de la barrière. L'anomalie à tension nulle de la diode $n$ Ge- $p$ GaAs est expliquée par une émission cohérente de phonons transverses acoustiques du Ge et une émission incohérente de phonons transverses acoustiques du GaAs. 